\title{
Histological Study of Expression of Seedlessness in Citrus kinokuni 'Mukaku Kishu' and Its Progenies
}

\author{
Atsu Yamasaki and Akira Kitajima ${ }^{1}$ \\ Graduate School of Agriculture, Kyoto University, 12-1 Hacchonawate, Takatsuki, Osaka 569-0096, Japan \\ Norihiro Ohara and Mitsutoshi Tanaka \\ Kochi Prefectural Agriculture Research Center, Tei 268, Asakura, Kochi, 780-8064, Japan
}

Kojiro Hasegawa

Faculty of Agriculture, Kochi University, Otsu 200, Monobe, Nankoku, Kochi 783-8502, Japan

\begin{abstract}
Additional INDEX words. abnormal embryo sac, embryo development, fertilization, pummelo, seed formation, seedless expression

ABstract. The factors of seedless expression in Citrus kinokuni hort. ex Tanaka 'Mukaku Kishu', 'Southern Yellow' \{'Tanikawa Buntan' pummelo [an uncertain hybrid with $C$. maxima (Burm.) Merr.] $\times$ 'Mukaku Kishu'\}, and the hybrid seedling BSY lines of 'Southern Yellow' $\times$ Bu1-7 pummelo [chance seedling of 'Suisho Buntan' pummelo $(C$. maxima)] were investigated histologically. 'Mukaku Kishu', 'Southern Yellow', BSY18, and BSY21 are completely seedless, whereas others are seedy. The percentage of abnormal embryo sacs and fertilization in seedless cultivars and lines showed no differences from seedy ones, indicating that the seedlessness of 'Mukaku Kishu' and its progenies was not involved in abnormal embryo sacs and unfertilized ovules. In seedy cultivars and lines, embryos developed to the cotyledon stage by 14 weeks after pollination. In the seedless cultivars and lines, however, embryos only developed to the early stages of zygote or embryo development. These results indicate that the seedless expression of the seedless progenies of 'Mukaku Kishu' coincides with 'Mukaku Kishu' and is caused by an arrested seed development at the early stage.
\end{abstract}

Seedlessness is one of the most important breeding objectives in citrus. Seedlessness is caused by several factors such as self-incompatibility, male or female sterility, early arrested seed after fertilization, and so on. However, self-incompatibility and male sterility cause fruit to produce seeds in crosspollination. Therefore, female sterility and early arrested seed are the most important characteristics for stable seedless expression. Female sterility includes abnormal embryo sac formation, such as no embryo sac formation or no egg cell in the embryo sac, and unfertilization derived from other reasons specific to the female organ system. We reported previously that the seedlessness of Hyuganatsu (Citrus tamurana hort. ex Tanaka) cv. Muroto Konatsu results from the inhibition of pollen tube growth into the pistil when cross-pollinated (Kitajima et al., 2001) and that 'Nankan No. 20' satsuma mandarin (C. unshiu Marc.) is involved in both abnormal embryo sacs and early arrested seed (Yamasaki et al., 2003).

'Kishu mikan' ( $C$. kinokuni), which has been a primary cultivar for more than 100 years in Japan, is seedy such as 'Hira Kishu' (C. kinokuni) except seedless 'Mukaku Kishu', which was caused by mutations. Although some seedless citrus are triploid (Soost and Cameron, 1969), 'Mukaku Kishu' was confirmed to be diploid (Befu et al., 2002). 'Mukaku Kishu' produces completely seedless fruit even if cross-pollinated (Iwamasa, 1978; Nesumi et al., 1992). Because a part of the progenies inherit the seedless characteristics (Nesumi et al., 2001), 'Mukaku Kishu' has received attention as a breeding parent to produce seedless cultivars. A new seedless cultivar, Southern Yellow, which originated from a cross between

Received for publication 2 Sept. 2006. Accepted for publication 28 June 2007. ${ }^{1}$ Corresponding author. E-mail: kitajima@kais.kyoto-u.ac.jp.
'Tanikawa Buntan' pummelo and 'Mukaku Kishu', has been released (Kobayashi, 1995; Yoshida et al., 2005a, 2005b).

Earlier studies of seedlessness in 'Mukaku Kishu' reported that pollen tube growth was inhibited after pollination, and seedless fruit was produced by fruit development resulting from the stimulation of pollen tube growth without fertilization (Iwasaki, 1953; Takahashi, 1967). Recently, Ishii et al. (1999) documented that fertilization occurred and the zygote or embryo was arrested in 'Mukaku Kishu', but the ratio of abnormal embryo sacs, no fertilization, and arrested zygote effects on seedless expression is still not clear. Furthermore, the consistency of seedless expression must be confirmed between 'Mukaku Kishu' and its progenies.

In this study, we histologically demonstrate the factors of seedless expression in 'Mukaku Kishu', 'Southern Yellow', and the hybrid seedlings of 'Southern Yellow' $\times$ pummelo.

\section{Materials and Methods}

'Hira Kishu', 'Mukaku Kishu', 'Tanikawa Buntan' pummelo, 'Southern Yellow', Bu1-7 pummelo, and eight seedlings (BSY1, BSY3, BSY17, BSY18, BSY19, BSY21, BSY27, and BSY29) of the BSY line (hybrid of Bu1-7 pummelo and 'Southern Yellow') were used for this study. These cultivars and lines were grown in the Fruit Tree Research Institute of Kochi Prefectural Agriculture Research Center, Kochi, Japan. BSY lines were crossing in 1994 and grafted on 34-year-old 'Nankan No. 4' satsuma mandarin (C. unshiu) interstock in 1995.

Five to 10 ovaries sampled at anthesis were dissected, and ovule numbers were counted under a light microscope. Flowers were hand pollinated with Hyuganatsu pollen on the flowering day. Each five to 10 ovaries or fruitlets were collected at 0,7 , 14,21 and $28 \mathrm{~d}$ after pollination (DAP) and fixed in FAA 
(5 formalin : 5 glacial acetic acid : 90 ethanol 70\%, v/v/v). Fixed samples were dehydrated by ethanol-butanol series and embedded into paraffin blocks. Sections were cut to a $6-\mu \mathrm{m}$ thickness with a rotary microtome and were double stained with acid fuchsin and fast green. Embryo sac development and fertilization were observed under a light microscope. Fertilization was estimated by the presence of free cell division in the embryo sac. The fertilization ratio was calculated as the number of fertilized ovules per number of ovules examined at 28 DAP. Ovules with an abnormal embryo sac were distinguished into three types defined by Kitajima et al. (1993): 1) embryo sac without trace, 2) no embryo sac with red-stained cell trace, and 3) an embryo sac with no nucleus. The percentage of abnormal embryo sacs was calculated as the sum of 0 to 21 DAP.

To observe embryo development after fertilization, each three to five fruits were collected 8 and 14 weeks after pollination (WAP), and the seeds were fixed in FAA. As the size of the seeds became clearly distinguishable at 14 WAP, they were visually grouped into developing seeds (large seeds) and others (small seeds); the seeds at 8 WAP were not grouped because of their similar size. The clear-staining procedure described later follows that of Herr (1974), Kojima et al. (1991) and Ishii et al. (1999). Fixed samples were soaked in $70 \%$ ethanol for 20 to $30 \mathrm{~min}$ and $10 \% \mathrm{KOH}$ for 2 to $3 \mathrm{~min}$. After being washed four times in distilled water, the seeds were dehydrated through $90 \%$ ethanol for 20 to 30 min and cleared in BB-4 1/2 fluid [mixture of 2 lactic acid : 2 chloral hydrate : 2 phenol : 2 clove oil : 1 xylem : 1 benzyl benzoate by weight (Herr, 1982)] overnight at room temperature. The cleared seeds were dissected, and their outer and inner integuments were removed by tweezers or fine needles under a light microscope. The naked nucellus with embryo sac was mounted on a glass slide filled with the clearing fluid. The glass slide was sealed with a coverslip. The developmental stage of the embryo was observed by microscope (BX50; Olympus Co., Tokyo) with
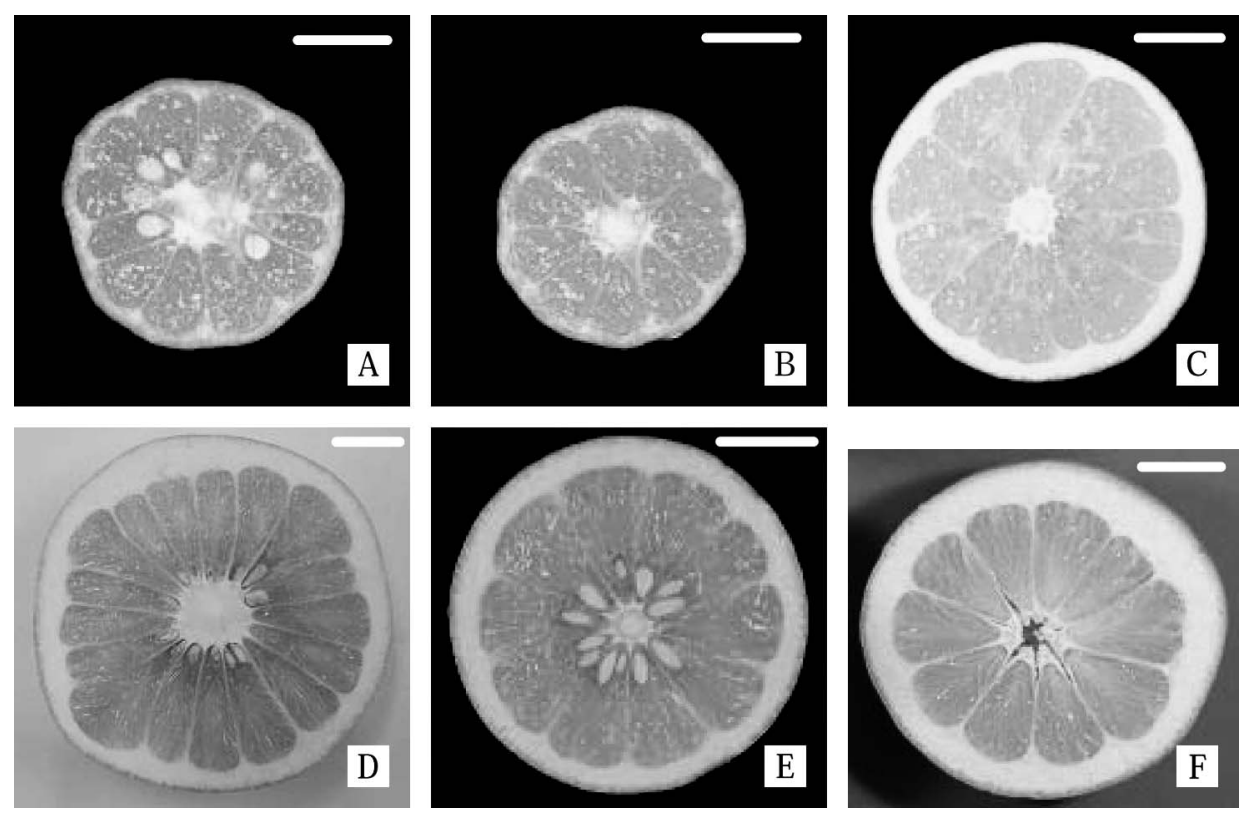

Fig. 1. (A-F) Fruit section in BSY lines [hybrid seedlings of 'Southern Yellow' (Citrus maxima 'Tanikawa Buntan' $\times$ Citrus kinokuni 'Mukaku Kishu') $\times$ C. maxima Bu1-7], their parents, and grandparents. (A) Citrus kinokuni 'Hira Kishu' (seedy). (B) 'Mukaku Kishu' (seedless). (C) 'Southern Yellow' (seedless). (D) Bu1-7 pummelo (seedy). (E) BSY1 (seedy). (F) BSY18 (seedless). Bars = $2.0 \mathrm{~cm}(\mathbf{A}-\mathbf{C}), 3.0 \mathrm{~cm}(\mathbf{D}-\mathbf{F})$. differential interference contrast optics. The sample, in which the interior of an embryo sac such as zygote or embryo was not able to observed, was evaluated as unfertilized ovules with the disappearance of the swelling of the embryo sac.

Two to 10 hand- and open-pollinated fruits were harvested at maturity, and fruit weight and seed numbers were recorded. Seeds were categorized visually as perfect seed (normal developing seed), imperfect seed (poorly developing seed), and ovulelike seed less than $3.0 \mathrm{~mm}$ long.

\section{Results and Discussion}

Number Of SEedS AND OvUles PER FRUIT. Unfertilized ovules from mature fruit were usually less than $3.0 \mathrm{~mm}$ long. However, undeveloped fertilized seeds in which the embryo degenerated during the early stages, such as in a zygote or proembryo, were also less than $3.0 \mathrm{~mm}$ long. Therefore, these fertilized seeds and unfertilized ovules less than $3.0 \mathrm{~mm}$ long were categorized as "ovulelike seed." Evaluation of seed number in 'Mukaku Kishu', 'Southern Yellow', BSY18, and BSY21 revealed that neither perfect nor imperfect seeds were found in them. Therefore, they are regarded as completely seedless cultivars and lines (Fig. 1, Table 1). The seed production characteristics are completely identical between 'Mukaku Kishu' and its seedless progenies. In the remaining cultivars and lines, the percentages of perfect and imperfect seeds to ovules per fruit were $23.1 \%$ to $67.4 \%$, indicating that these are seedy cultivars and lines.

More than 50 ovules per ovary were observed in the BSY lines and the 'Tanikawa Buntan' pummelo (Table 1). The number of seeds did not correspond to the number of ovules in the BSY lines. The number of ovules per ovary in seedless BSY18 and BSY21 was 67.2 and 54.8 respectively, which was not different from other seedy BSY lines. The number of ovules per ovary in seedless 'Southern Yellow' was not different from seedy Bu1-7 pummelo, whereas the number in seedless 'Mukaku Kishu' was less than that of seedy 'Hira Kishu.' In citrus, the difference in locule numbers per fruit and ovule numbers per locule has been considered to cause an increase in seed number (Tanaka, 1948). Kitajima et al. (1997) reported that the difference in seed numbers between 'Tosa Buntan' pummelo (C. maxima) and 'Suisho Buntan' pummelo reflected the difference of ovules formed. In this study, locule numbers per ovary, ovule numbers per locule, and locule numbers per ovary were not different between seedless and seedy cultivars and lines. These observations suggested that the number of locules and ovules is not involved in the seedless characteristics of 'Mukaku Kishu'.

Embryo SAC FORMATION AND FERTILIZATION. In all cultivars and lines except for 'Hira Kishu' and 'Mukaku Kishu', the embryo sac at anthesis fully developed to the stage 


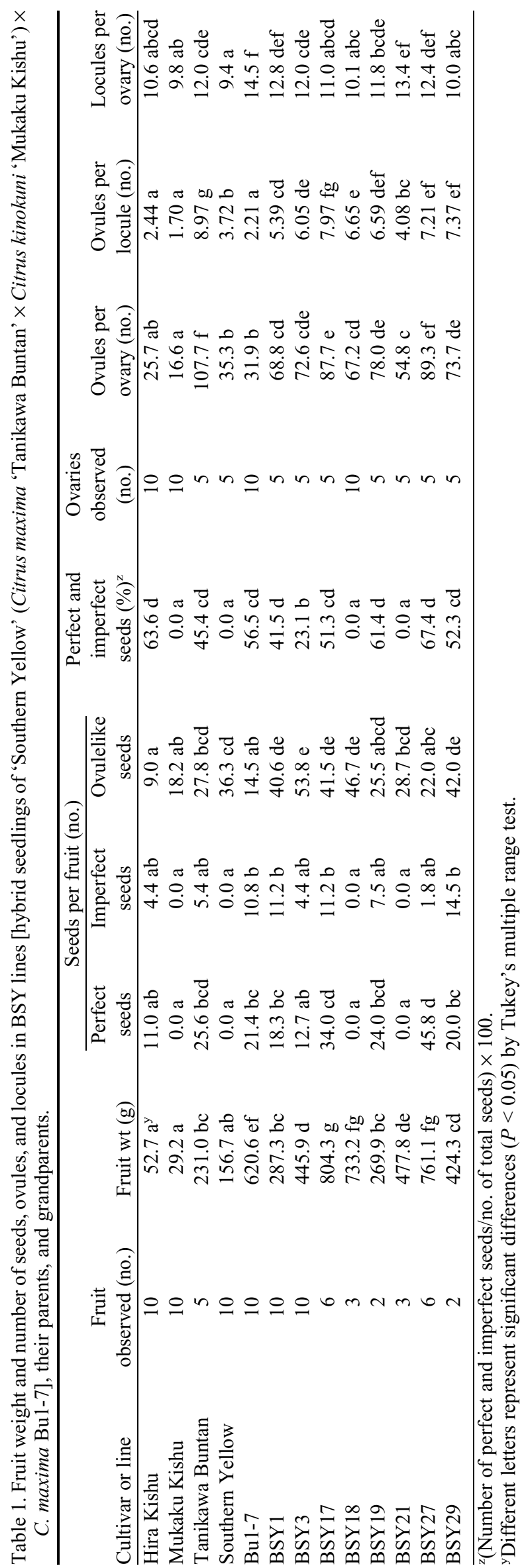

of one to eight nuclei. Full developed embryo sacs were also observed in seedless BSY18 and BSY21 (Table 2). In 'Hira Kishu' and 'Mukaku Kishu', many ovules were still embryo sac mother cell, and the embryo sac development was slower than others. Despite differences in embryo sac development, the embryo sacs of all cultivars and lines were full developed at 7 DAP (data not shown). Some abnormal embryo sacs were observed from anthesis, and the percentage of abnormal embryo sacs was calculated by using the samples of 0 to 21 DAP. The appearance of an abnormal embryo sac is also thought to be an important factor for seedless expression. In this study, the highest percentage of abnormal embryo sacs was $23.4 \%$ in seedy Bu1-7 pummelo (Table 3). The percentage of abnormal embryo sacs in seedy cultivars and lines ranged from $5.2 \%$ to $15.0 \%$, except in Bu1-7 pummelo. The values in seedless 'Mukaku Kishu', 'Southern Yellow', BSY18, and BSY21 were also about $10 \%$, which is not higher than those of seedy ones. In grape (Vitis vinifera L.), Wang and Horiuchi (1990) reported that most embryo sacs lacked egg cells or synergids or exhibited degeneration of the egg apparatus in the seedless cultivar Himrod Seedless. Therefore, the ratio of abnormal embryo sac is an important factor for seedless expression. In Citrus, Yamasaki et al. (2003) reported that the percentage of abnormal embryo sacs in the seedless 'Nankan No. 20 ' satsuma mandarin was about $25 \%$, higher than in seedy 'Tosa Buntan' and 'Suisho Buntan' pummelo, which was about $5 \%$ to $10 \%$. In this study, the percentage of abnormal embryo sac in seedless cultivars and lines was almost equal to that in seeded ones. We conclude that seedless expression of 'Mukaku Kishu' and its progenies is not involved in abnormal embryo sacs.

These observations demonstrated that fertilization is one of the most important factors for seedless expression. In this study, all fertilization occurred 14 DAP (Table 3 ). The ratio of fertilized ovules was high in seedy 'Hira Kishu' (Fig. 2A), 'Tanikawa Buntan' pummelo, and BSY27, but low in seedy BSY3. The ratio of fertilization in seedy cultivars and lines ranged from $43.8 \%$ to $97.9 \%$. The ratio of fertilization in seedless cultivars and lines ranged from $63.0 \%$ to $75.0 \%$ (Fig. $2 \mathrm{~B}, \mathrm{C}$ ), which was not lower than seedy ones. It has been reported that no fertilized ovule was observed in seedless 'Muroto Konatsu', a mutant of seedy Hyuganatsu, because pollen tube growth in the style was completely suppressed, and the percentage of fertilized ovules was about $60 \%$ with permission of pollen tube growth in the common seedy type (Kitajima et al., 2001). So, a certain female system such as suppression of compatible pollen tube growth in the style of 'Muroto Konatsu' may be a factor for seedless expression. In this study, however, the fertilization occurred in 'Mukaku Kishu' and its seedless progenies to the same degree in seedy cultivars and lines. The result suggested that the pollen growth was not inhibited in the style of 'Mukaku Kishu' and its seedless progenies. These observations indicated that the unfertilized ovules are not involved in the seedless characteristics of 'Mukaku Kishu'.

EMbryo deVelopMent. In this study, the embryo stages of zygote and proembryo, and embryo sac degeneration were mainly observed at 8 WAP (Fig. 2D-F, Table 4). Endosperm nuclei were observed in the embryo sacs in which zygotes and proembryos existed (data not shown). Not all interior embryo sacs of degenerated ovules could be observed. In Bu1-7 pummelo, with embryo development that was very fast, 
Table 2. Percentage of ovules in different embryo sac developmental stages and abnormal embryo sacs at anthesis in BSY lines [hybrid seedlings of 'Southern Yellow' (Citrus maxima 'Tanikawa Buntan' $\times$ Citrus kinokuni 'Mukaku Kishu') $\times$ C. maxima Bu1-7], their parents, and grandparents.

\begin{tabular}{|c|c|c|c|c|c|c|c|c|c|c|}
\hline \multirow{2}{*}{ Cultivar or line } & \multirow{2}{*}{$\begin{array}{c}\text { Ovules } \\
\text { observed } \\
\text { (no.) }\end{array}$} & \multicolumn{8}{|c|}{ Stages of embryo sac development (\%) } & \multirow{2}{*}{$\begin{array}{c}\text { Abnormal } \\
\text { embryo } \\
\operatorname{sac}^{\mathrm{z}}(\%)\end{array}$} \\
\hline & & $\begin{array}{l}\text { Megaspore } \\
\text { mother cell }\end{array}$ & Dyad & Tetrad & \multicolumn{4}{|c|}{ Embryo sac nuclei } & $\begin{array}{c}\text { Mature } \\
\text { embryo sac }\end{array}$ & \\
\hline Hira Kishu & 17 & 58.8 & 11.8 & 0.0 & 23.5 & 0.0 & 0.0 & 0.0 & 0.0 & 5.9 \\
\hline Tanikawa Buntan & 52 & 0.0 & 0.0 & 0.0 & 3.8 & 26.9 & 38.5 & 26.9 & 0.0 & 3.9 \\
\hline Southern Yellow & 32 & 0.0 & 0.0 & 0.0 & 6.3 & 34.4 & 46.9 & 6.2 & 0.0 & 6.2 \\
\hline Bu1-7 & 25 & 0.0 & 0.0 & 0.0 & 12.0 & 28.0 & 36.0 & 0.0 & 0.0 & 24.0 \\
\hline BSY3 & 31 & 0.0 & 0.0 & 0.0 & 3.2 & 48.4 & 6.5 & 16.1 & 0.0 & 25.8 \\
\hline BSY17 & 41 & 0.0 & 0.0 & 0.0 & 39.0 & 31.7 & 2.5 & 0.0 & 0.0 & 26.8 \\
\hline BSY18 & 41 & 0.0 & 0.0 & 0.0 & 4.9 & 22.0 & 26.8 & 24.4 & 7.3 & 14.6 \\
\hline BSY19 & 11 & 0.0 & 0.0 & 0.0 & 0.0 & 9.1 & 54.5 & 18.2 & 0.0 & 18.2 \\
\hline BSY21 & 25 & 0.0 & 0.0 & 0.0 & 0.0 & 12.0 & 36.0 & 32.0 & 0.0 & 20.0 \\
\hline BSY27 & 20 & 0.0 & 0.0 & 0.0 & 0.0 & 15.0 & 30.0 & 30.0 & 25.0 & 0.0 \\
\hline
\end{tabular}

${ }^{\mathrm{z}}$ Abnormal embryo sac was determined according to the manner used by Kitajima et al. (1993): $1=$ embryo sac without trace, $2=$ no embryo sac with red cell trace, and $3=$ an embryo sac with no nucleus.

$23.0 \%$ of the ovules developed to the globular stage, and the most developed stage was torpedo embryos at 8 WAP.

At 14 WAP, in all seedy cultivars and lines except for BSY19 and BSY29, cotyledon stage embryos were observed in large seeds (Fig. 2G, Table 5). In 'Tanikawa Buntan' pummelo and BSY3, not only cotyledon stage but also proembryo or globular to torpedo stages were observed. The embryo development of BSY19 and BSY29 was delayed and the embryos were only developed to the globular stage. Small seeds in seedy cultivars and lines involved many degenerated ovules/seed and fertilized seeds from which embryos developed to the heartshaped stage in maximum. Seedless cultivars and lines had no large seeds, only small ones. Small seeds of seedless cultivars and lines except 'Southern Yellow' contained fertilized ones with embryos that were in zygote and proembryo stages (Fig. $2 \mathrm{H}$, Table 5). Developmental stages of embryo among seedless cultivars and lines were similar between 8 and 14 WAP. However, the embryos of 'Southern Yellow' were developed until the heart-shaped or globular stages, in contrast to other seedless ones (Fig. 2I). In seedless grapes of seedless expression 'Thompson Seedless' (V. vinifera) (Pearson, 1933), 'Monukka' (V. vinifera) and 'Mukaku Siro' (V. vinifera) (Wang et al., 1993) were involved in arrested embryo after fertilization. Thus, an arrested embryo after fertilization is an important factor for seedless expression. Ishii et al. (1999) reported that the zygote of fertilized ovules in 'Mukaku Kishu' divided into two or more cells but did not develop to globular embryo at 14 WAP. The results from our experiment indicate that embryo development of 'Mukaku Kishu' and its progenies except 'Southern Yellow' is clearly inhibited and arrested until at least 8 WAP, which agrees with the results of 'Mukaku Kishu' by Ishii et al. (1999). Although the embryo development of

Table 3. Percentage of abnormal embryo sacs and fertilized ovules in BSY lines [hybrid seedlings of 'Southern Yellow' (Citrus maxima 'Tanikawa Buntan' $\times$ Citrus kinokuni 'Mukaku Kishu') $\times$ C. maxima Bu1-7], their parents, and grandparents.

\begin{tabular}{|c|c|c|c|c|c|c|c|c|c|}
\hline \multirow{3}{*}{$\frac{\text { Cultivar or line }}{\text { Hira Kishu }}$} & \multirow{3}{*}{ 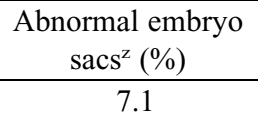 } & \multicolumn{8}{|c|}{ Fertilized ovules $(\%)^{y}$} \\
\hline & & \multicolumn{2}{|c|}{7 DAP } & \multicolumn{2}{|c|}{14 DAP } & \multicolumn{2}{|c|}{$21 \mathrm{DAP}$} & \multicolumn{2}{|c|}{28 DAP } \\
\hline & & 0.0 & $(20)$ & 20.9 & $(50)$ & 57.4 & (78) & 94.7 & $(38)$ \\
\hline Mukaku Kishu & 10.7 & 0.0 & (10) & 32.4 & (41) & 34.3 & $(102)$ & 64.3 & (42) \\
\hline Southern Yellow & 11.5 & 0.0 & (28) & 31.3 & $(63)$ & 60.5 & (75) & 63.0 & (81) \\
\hline Bu1-7 & 23.4 & 0.0 & (13) & 23.5 & (50) & 64.3 & (50) & 78.6 & (42) \\
\hline BSY1 & 9.3 & 0.0 & (34) & 26.3 & (90) & 37.4 & $(103)$ & 72.3 & (100) \\
\hline BSY17 & 11.6 & 0.0 & (54) & 30.9 & (113) & 58.8 & $(90)$ & 62.3 & (115) \\
\hline BSY18 & 10.5 & 0.0 & (10) & 62.0 & (106) & 79.3 & (66) & 75.0 & (36) \\
\hline BSY19 & 17.6 & 0.0 & (10) & 30.8 & (61) & 68.9 & (54) & 76.9 & (52) \\
\hline BSY21 & 10.0 & 0.0 & (10) & 41.2 & (17) & & & & \\
\hline BSY27 & 5.3 & 0.0 & (34) & 87.5 & (10) & 100.0 & (10) & 91.7 & (12) \\
\hline BSY29 & 9.3 & 0.0 & (15) & 36.8 & (25) & 92.9 & (30) & & \\
\hline
\end{tabular}

${ }^{\mathrm{z}}$ Abnormal embryo sac was shown in Table 2. Sum of 0 to 21 DAP.

${ }^{\mathrm{y}}$ Fertilization was estimated by the presence of free cell division in the embryo sac. Parentheses in table represent number of ovules observed.

"Not examined.

DAP, d after pollination. 

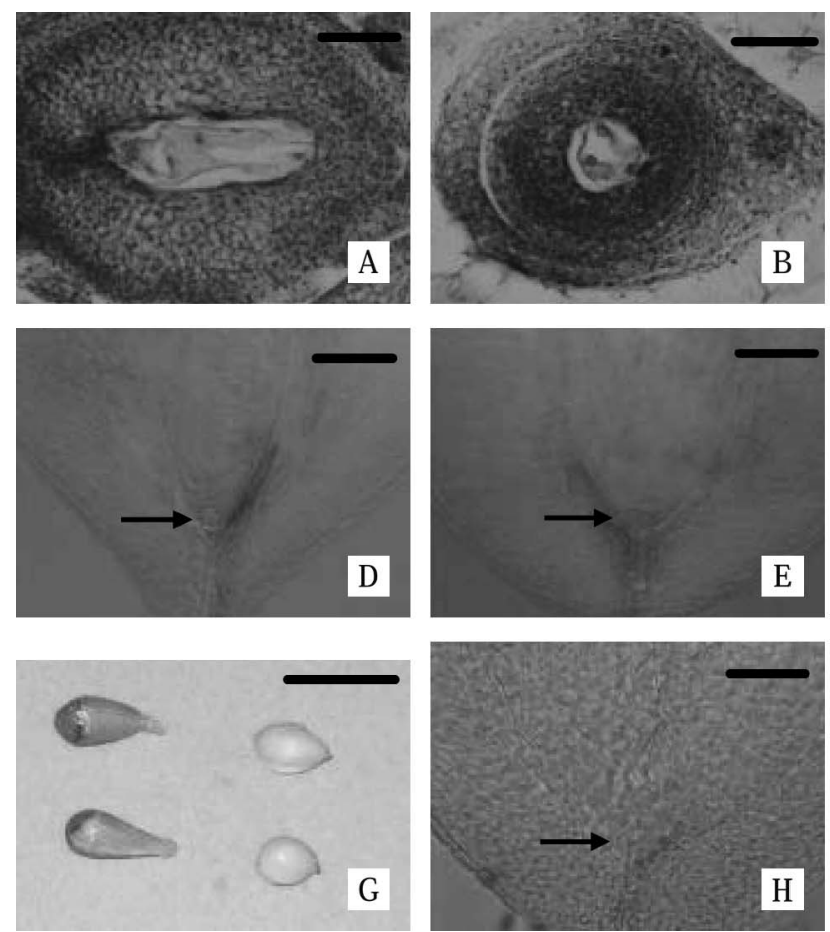

Fig. 2. (A-I) Development of the embryo sac and embryo in Citrus kinokuni 'Hira Kishu' (A, D, G), C. kinokuni 'Mukaku Kishu' (B, E, H), and 'Southern Yellow' (Citrus maxima 'Tanikawa Buntan' $\times$ 'Mukaku Kishu') (C, F, I). (A-C) Fertilization at $28 \mathrm{~d}$ after pollination. (D-F) Zygote stage (arrow) at 8 weeks after pollination (WAP). (G) Cotyledon stage (right) and seed (left) at 14 WAP. (H) Zygote stage (arrow) at 14 WAP. (I) Heartshaped stage at 14 WAP. Bars $=60 \mu \mathrm{m}(\mathbf{A}-\mathbf{F}), 7.0 \mathrm{~mm}(\mathbf{G}), 60 \mu \mathrm{m}(\mathbf{H}), 120 \mu \mathrm{m}(\mathbf{I})$.

'Southern Yellow' was promoted to the heart-shaped stage more than other seedless ones, it was clearly inhibited compared with seedy ones. These observations suggested that seedless expression in 'Mukaku Kishu' and its seedless progenies are involved in embryo degeneration or developmental arrest during the early stage. In contrast, the embryos of seedy BSY19 and BSY29 only developed to the proembryo and globular stages at 14 WAP, and the other seedy cultivars and lines developed to the cotyledon stage. It is unclear whether the
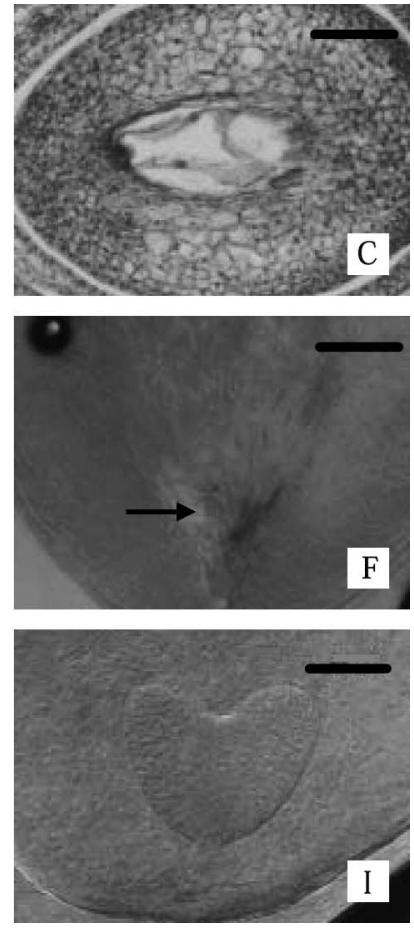

differences in embryo development were caused by genetic or environmental factors.

This study clarified the following: 1) the seedless expression of the seedless progenies of 'Mukaku Kishu' coincides with 'Mukaku Kishu', 2) most ovules in 'Mukaku Kishu' and seedless progenies inhibit zygote or embryo development at early stages, and 3 ) the expression of seedlessness derived from 'Mukaku Kishu' is caused by one factor of early arrested seed.

The clarification of the number of seedless expression factors of 'Mukaku Kishu' is one crucial development for DNA markers, which is linked to its seedless characteristics for earlier selection of the seedless seedlings of its progenies. Nesumi et al. (2001) reported that the ratios of seedless to seedy seedlings were $1: 1$ in the progenies of 'Mukaku Kishu' $\times$ some seedy mandarins \{'Kiyomi' tangor [C. unshiu $\times C$. sinensis (L.) Osbeck], 'Lee' mandarin $[C$. clementina hort. ex Tanaka $\times$ (C. paradisi Macfad. $\times$ C. tangerina hort. ex Tanaka)], and 'King' mandarin (C. nobilis Lour.) $\}$ and 1:3 in the progeny of 'Mukaku Kishu' $\times$ pummelo, 'Hirado Buntan' pummelo (C. maxima) and 'May Pummelo' pummelo $(C$. hassaku hort. ex Tanaka $\times$ 'Hirado Buntan' pummelo). Moreover, they proposed that the seedless characteristics derived from 'Mukaku Kishu' were controlled by a dominant gene $(F s)$ and a repressor gene (IS) that dominantly inhibits seedless expression. Supposed genotypes of 'Mukaku Kishu', these seedy mandarins and pummelos were $F s f_{s}$-isis, $f_{S} f_{s}$-isis, and $f_{s} f_{s-I s i s}$ respectively. This hypothesis shows that seedless expression derived from

Table 4. Percentage of seeds in different embryo developmental stages at 8 weeks after pollination in BSY lines [hybrid seedlings of 'Southern Yellow' (Citrus maxima 'Tanikawa Buntan' $\times$ C. kinokuni 'Mukaku Kishu') $\times$ C. maxima Bu1-7], their parents, and grandparents.

\begin{tabular}{|c|c|c|c|c|c|c|c|c|}
\hline \multirow[b]{2}{*}{ Cultivar or line } & \multirow{2}{*}{$\begin{array}{c}\text { Seeds } \\
\text { observed } \\
\text { (no.) }\end{array}$} & \multicolumn{7}{|c|}{ Developmental stages (\%) } \\
\hline & & $\begin{array}{c}\text { Cotyledon } \\
\text { shaped }\end{array}$ & $\begin{array}{c}\text { Torpedo } \\
\text { shaped }\end{array}$ & $\begin{array}{l}\text { Heart } \\
\text { shaped }\end{array}$ & Globular & Proembryo & Zygote & Degeneration \\
\hline Hira Kishu & 26 & 0.0 & 0.0 & 0.0 & 0.0 & 46.2 & 19.2 & 34.6 \\
\hline Tanikawa Buntan & 48 & 0.0 & 0.0 & 0.0 & 0.0 & 10.9 & 41.3 & 47.8 \\
\hline Southern Yellow & 41 & 0.0 & 0.0 & 0.0 & 0.0 & 28.1 & 34.4 & 37.5 \\
\hline Bu1-7 & 35 & 0.0 & 7.7 & 7.7 & 30.8 & 3.8 & 0.0 & 50.0 \\
\hline BSY3 & 50 & 0.0 & 0.0 & 0.0 & 0.0 & 4.4 & 41.3 & 54.3 \\
\hline BSY17 & 100 & 0.0 & 0.0 & 0.0 & 0.0 & 18.1 & 55.4 & 26.5 \\
\hline BSY18 & $-^{z}$ & - & - & - & - & - & - & - \\
\hline BSY19 & $-^{z}$ & - & - & - & - & - & - & - \\
\hline BSY21 & 30 & 0.0 & 0.0 & 0.0 & 0.0 & 0.0 & 33.3 & 66.7 \\
\hline BSY27 & 70 & 0.0 & 0.0 & 0.0 & 0.0 & 31.1 & 53.4 & 15.5 \\
\hline
\end{tabular}

${ }^{\mathrm{z}}$ Not examined. 
Table 5. Percentage of seeds in different embryo developmental stages at 14 weeks after pollination in BSY lines [hybrid seedlings of 'Southern Yellow' (Citrus maxima 'Tanikawa Buntan' $\times$ C. kinokani 'Mukaku kishu') $\times$ C. maxima Bul-7], their parents, and grandparents.

\begin{tabular}{|c|c|c|c|c|c|c|c|c|}
\hline \multirow[b]{2}{*}{ Cultivar or line } & \multirow{2}{*}{$\begin{array}{c}\text { Seeds } \\
\text { observed } \\
\text { (no.) }\end{array}$} & \multicolumn{7}{|c|}{ Developmental stages (\%) } \\
\hline & & $\begin{array}{c}\text { Cotyledon } \\
\text { shaped }\end{array}$ & $\begin{array}{c}\text { Torpedo } \\
\text { shaped }\end{array}$ & $\begin{array}{c}\text { Heart } \\
\text { shaped }\end{array}$ & Globular & Proembryo & Zygote & Degeneration \\
\hline \multicolumn{9}{|l|}{ Large seeds } \\
\hline Mukaku Kishu & $-^{z}$ & - & - & - & - & - & - & - \\
\hline Tanikawa Buntan & 12 & 25.0 & 25.0 & 25.0 & 25.0 & 0.0 & 0.0 & 0.0 \\
\hline Southern Yellow & $-^{z}$ & - & - & - & - & - & - & - \\
\hline BSY1 & 12 & 100.0 & 0.0 & 0.0 & 0.0 & 0.0 & 0.0 & 0.0 \\
\hline BSY3 & 13 & 66.7 & 8.3 & 8.3 & 8.3 & 8.3 & 0.0 & 0.0 \\
\hline BSY17 & 10 & 100.0 & 0.0 & 0.0 & 0.0 & 0.0 & 0.0 & 0.0 \\
\hline BSY18 & $-^{z}$ & - & - & - & - & - & - & - \\
\hline BSY19 & 9 & 0.0 & 0.0 & 0.0 & 11.1 & 55.6 & 11.1 & 22.2 \\
\hline BSY21 & $-^{z}$ & - & - & - & - & - & - & - \\
\hline Hira Kishu & 8 & 0.0 & 10.0 & 0.0 & 10.0 & 0.0 & 10.0 & 70.0 \\
\hline Mukaku Kishu & 7 & 0.0 & 0.0 & 0.0 & 0.0 & 50.0 & 33.3 & 16.7 \\
\hline Tanikawa Buntan & 10 & 0.0 & 0.0 & 0.0 & 10.0 & 20.0 & 0.0 & 70.0 \\
\hline Southern Yellow & 27 & 0.0 & 0.0 & 15.4 & 23.1 & 3.8 & 7.7 & 50.0 \\
\hline Bu1-7 & 8 & 52.9 & 0.0 & 0.0 & 0.0 & 0.0 & 0.0 & 47.1 \\
\hline BSY1 & 9 & 0.0 & 0.0 & 20.0 & 26.7 & 13.3 & 0.0 & 40.0 \\
\hline BSY3 & 7 & 0.0 & 0.0 & 0.0 & 0.0 & 0.0 & 0.0 & 100.0 \\
\hline BSY17 & 10 & 0.0 & 0.0 & 0.0 & 42.1 & 10.5 & 0.0 & 47.4 \\
\hline BSY18 & 28 & 0.0 & 0.0 & 0.0 & 0.0 & 0.0 & 14.3 & 85.7 \\
\hline BSY19 & 10 & 0.0 & 0.0 & 0.0 & 0.0 & 20.0 & 0.0 & 80.0 \\
\hline BSY21 & 10 & 0.0 & 0.0 & 0.0 & 0.0 & 33.3 & 33.3 & 33.3 \\
\hline
\end{tabular}

'Mukaku Kishu' involves two factors. However, it is speculated that $F s$ is a gene associated with the inhibition of early zygote and embryo development, which was one factor for seedlessness expression in this study. Further investigation must be conducted to explain the degeneration or developmental arrest of the zygote and embryo, and to clarify whether the phenotype is derived from the seed parent or zygote genotype.

\section{Literature Cited}

Befu, M., A. Kitajima, and K. Hasegawa. 2002. Classification of the citrus chromosomes with same types of chromomycin A banding patterns. J. Jpn. Soc. Hort. Sci. 71:394-400. (In Japanese with English summary).

Herr, J.M., Jr. 1974. A clearing squash technique for the study of ovule and megagametophyte development in angiosperms, p. 230-235. In: A.E. Raford, W.C. Dickison, J.R. Massey, and C.R. Bell (eds.). Vascular plants. Harper and Row, New York.

Herr, J.M., Jr. 1982. An analysis of methods for permanently mounting ovules cleared in four-and-a-half type clearing fluids. Stain Technol. 57:161-169.

Ishii, K., H. Nesumi, T. Takagi, and T. Hirabayashi. 1999. Histological observation on seedlessness in 'Mukaku-Kishu' (C. kinokuni hort. ex Tanaka). J. Jpn. Soc. Hort. Sci. 68(Suppl. 2):173. (abstr.) (in Japanese).

Iwamasa, M. 1978. Kankitsu no ikushu nikansuru shomondai 19. Agr. Hort. 53:105-108. (in Japanese).
Iwasaki, T. 1953. Shurui narabini hinshu, p. 138-140. In: T. Iwasaki (ed.). Kankitsu saibai yosetsu. Yokendo, Tokyo. (in Japanese).

Kitajima, A., A. Kataoka, and K. Hasegawa. 1997. Differences of fertilization and seed formation between 'Tosa-Buntan' and 'SuishoBuntan' pummelo (Citrus grandis [L.] Osb.). J. Jpn. Soc. Hort. Sci. 66(Suppl. 1):110-111. (abstr.) (in Japanese).

Kitajima, A., Y. Okada, and K. Hasegawa. 2001. Pollen tube growth, fertilization and seed development in Hyuganatsu (Citrus tamurana) seedless cultivars 'Muroto-Konatsu' and 'Nishiuchi-Konatsu'. J. Jpn. Soc. Hort. Sci. 70:320-327 (in Japanese with English summary).

Kitajima, A., T. Uchida, and M. Ishida. 1993. Influence of embryo-sac development and fertilization processes on seed formation of japanese persimmon (Diospyros kaki L. f.) cv. Fuyu (in Japanese with English summary). J. Jpn. Soc. Hort. Sci. 62:327-335.

Kobayashi, S. 1995. Sinhinshu no saibaigizyutsu. Kazitsu Nihon 50:101 (in Japanese).

Kojima, A., K. Hinata, and S. Noda. 1991. An improvement of squash method for the cytological study of female meiosis in Allium tuberosum, Liliaceae. Chromosome Info. Serv. 50:5-7.

Nesumi, H., Y. Ito, T. Yoshioka, and T. Yoshida. 1992. Female sterility of 'Mukakukishu' and its inheritance. J. Jpn. Soc. Hort. Sci. 61(Suppl. 1):36-37. (abstr.). (in Japanese).

Nesumi, H., M. Nakano, and T. Yoshida. 2001. Mode of inheritance on the abnormal development of impregnated ovules derived from Mukaku-kishu. J. Jpn. Soc. Hort. Sci. 70(Suppl. 2):403. (abstr.). (in Japanese).

Pearson, H.M. 1933. Parthenocarpy and seed abortion in Vitis vinifera. Proc. Amer. Soc. Hort. Sci. 29:169-175. 
Soost, R.K. and J.W. Cameron. 1969. Tree and fruit characters of Citrus triploid from tetraploid by diploid cross. Hilgardia 39:569579.

Takahashi, I. 1967. Shurui to hinshu, p. 49-50. In: I. Takahashi (ed.). Kankitsu. Yokendo, Tokyo. in Japanese.

Tanaka, Y. 1948. Kankitsurui no shuyokeishitsu, p. 25-33. In: Y. Tanaka (ed.). An iconograph of Japanese citrus fruits, vol. 1. Yokendo, Tokyo. (in Japanese).

Yamasaki, A., A. Kitajima, and K. Hasegawa. 2003. Histological study on female sterility in satsuma mandarin (C. unshiu Marc.). ChugokuShikoku Br Jpn. Soc. Hort. Sci. 42:10 (abstr.) (in Japanese).

Yoshida, T., H. Nesumi, T. Yoshioka, Y. Ito, I. Ueno, and Y. Yamada. 2005a. 'Kankitsu Chukanbohon Nou 5 Gou' ('Citrus Parental Line Norin No. 5') is useful for breeding seedless and early maturing cultivars. Bul. Natl. Inst. Fruit Tree Sci. 4:47-52 (in Japanese with English summary).

Yoshida, T., H. Nesumi, T. Yoshioka, Y. Ito, M. Yano, M. Nakano, I. Ueno, Y. Yamada, K. Ogawa, S. Murase, F. Takishita, T. Hidaka, and S. Kawai. 2005b. 'Kankitsu Chukanbohon Nou 6 Gou' ('Citrus Parental Line Norin No. 6') is useful for breeding seedless and functional component-rich cultivars. Bul. Natl. Inst. Fruit Tree Sci. 4:53-59 (in Japanese with English summary).

Wang, J. and S. Horiuchi. 1990. A histological study on the seedlessness in 'Himrod Seedless' grape. J. Jpn. Soc. Hort. Sci. 59:455-462. (in Japanese with English summary).

Wang, J., S. Horiuchi, and H. Matsui. 1993. A histological study of seedlessness in seedless grapes. J. Jpn. Soc. Hort. Sci. 62:1-7. (in Japanese with English summary). 